aseptic state of the urine. The operation was performed about sixteen and a half hours from the time the man was hit.

\section{Case XXIr.}

The second bladder wound was that of a man shot through the right buttock transversely; also by a shrapnel ball. Ad. mitted with extensive extravasation of blood and urine around the perineum and both buttocks. Opened up area of extravasathe perineum and both buttocks. Opened up area of extravasarectal fossa found the bullet, which practically dropped into one's hand. Owing to the man's state, which was very bad, no attempt was made to hunt for the wound of the bladder. The bladder was opened suprapubically and drained.

He was lept in hospital six days after operation; the perineum was then dry and doing well.

Perhaps this man should have been operated npon through the perineum by an external urethrotomy high up, but it was thought at the time that the suprapubic drain was the safest.

B.yYonet Wounds.

Only one bayonet wound was admitted to this hospital. This seems very surprising, as bayonet attacks wèc reported to be occurring frequently and one would have expected serious wounds.

\section{CASE XXIII.}

Private F. (Royal Fusiliers). Brought in urgentls by motor ambulance for treatment for severe haemorrhage from mouth and eye. The bayonet had entered at the inner canthus of the left eve and penetrated through the left maxillary antrum. He was bleeding freely from vessel round palatal and maxillary regions. Under ether the antrum was opened through the canine fossa, and the opening was rapidly enlarged. It was found full of blood soaked in clot. It was washed ont and packed in gauze soaked in hydrogen peroxide.

The ereball had escaped injur, evidently pushed aside by the baronet.

\section{Wouxds of Thorax.}

A considerable number of officers and men rvere almitted with perforating wounds of the thorax. Wounds of the upper part of the chest invariably did well; wounds of the lower part were serious and often fatal.

A short history of one case will illustrate the others.

\section{Case xxiv.}

Captain S., R.A.M.C., attached to the - Regiment, was shot through both lungs by a bullet while gallantly attending to wounded men under a heavy fire. For this act he has been awarded the Military Cross. He was stooping forward at the time with both arms extended away from the body. The bullet struck him about the lower part of the right axilla and emerged about the posterior axillary line of the left side. He was admitted with severe shock and dyspnoea. He had frequent cough, which he tried to prevent owing to the pain it produced. When put to bed and heroin 1 grain placed under the tongue he became cosier and the shock sradually disappeared The became casier and the shock gradually disappeared. The cough eased off and respiratory excursions became more marked; temperature normal, pulse rapid. There was marked dulluess over the right lung base. The breath sounds were vished to get up. In fact he did get up once or twice when the

At the end of the third day the temperature ran up to $103^{\circ} \mathrm{F}$. Dyspnoea became marked, and pain in breathing so severe that th hypodermic of morphine was given; the pain was all over the right base. Briefly, the course of events was: pleurisy of right base, pneumonia over the course of bullet wound through right lung, expectoration of old blood clots, high temperature. The left Iung, curiously enough, escaped, although there was dullness due to haemothorax of left base. The after-histor. was a slow recovery.

All the penetrating bullet wounds-those with entrance and exit wounds-behaved in a similar way. First, a period of dyspnoea, very little hacmoptysis, with shock slight or severe. Then a period, usually lasting for two or three days, of comparative quiet, with slightly quickened breathing, no haemoptysis, occasional cough, no rise of temperature. Then a rise of temperature, frequent and painful cough, with expectoration of particles of old blood clot, and thick mucus, and signs of pneumonia round the track of the bullet, and generally pleurisy of base from the haemothorax. The above only refer's to the course taken at the time of the wound and for some days afterwards. The after-history of these perforating wounds of the thorax often presents other features.

Local Anaesthesia.

Iocal anaesthesia on active service is a subject that will bear some study. The circumstances in which this form of anaesthesia can be applied are many. The condition of the wounded, owing to shock, haemorrhage, exposure, and gangrene, is often so serious that any operation, howerer small, entails formidable risks. For the prerention of shock, local anaesthesia has proved to be invaluable. It can abolish pain and so remore a powerful factor pro. ducing shock. It enables one to operate on mary patients when a general anaesthetic would be hazardous.

The principles of infiltration anaesthesia and of conduc. tion anaesthesia are easily mastered. One need only have a sound linowledge of the anatomy of the nerrous system. The surgeon must accustom himself to the needles and apparatus. Conduction and infiltration anaesthesis was carried ont in a large number of cases in this hospital. 'The method was employed in all the trephine cases except one. It was used in amputations of finger's and in cleaning up wounds in the hands and fingers. it was employed in operations on the toes, feet, ankle, leg, fractured thighs, opening a frontal sinus, removing contents of orbits, removing testicles, in shell wounds of limbs.

I hope to record these cases in a special article at a later date. The syringes and needles are not supplied by the army. One must bring one's own outfit. It can be conreniently packed in a light metal box enclosed in a canvas bag with a handle, Barker's infiltration needles are necessary, and also two sets of needles and syringes for the conduction anaesthesia. My outfit included also adrenalin in solution, beta eucaine in powders, and tablets of novocain-suprarenin. These tablets are added to physiologic salt solution, and can be made un to various strengths, as 0.5 per cent., 1 per cent. or 2 per cent. 'The tablets contain novocain and suprarenal extract. The solution can be boiled after adding a few drops of dilute hydrochloric acid, and so rendered quite sterile.

\section{ACIDOSIS AND THE NITROGEN PARTITION IN PREGNANCY.}

BY H. LFITH MURRAY, M.1.,

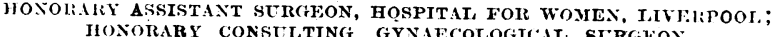

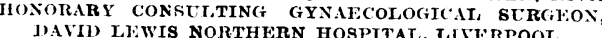

To Dr. Whitridge Williams is due the institution of a scientific examination of the urine in pregnancy. Recently he has modified to some considerable extent his origina! view that an ammonia coefficient over 10 to 15 per cent. is an indication for the prompt termination of pregnancy. Most of those working at the subject were more or less prepared for this change, but perhaps not all are yet con. vinced that his present view is a clear and sound one. It will be of interest, then, and possibly' of service, to review the later aspects of the question and to report some recent results in a series of cases.

It appear's to me regrettable that inrestigation, even in toxic cases, in maternity hospitals is so often limited to a quantitative estimation of the albumin, of the nrea, and occasionally of the ammonia, and to a qualitatice examination for acetone bodies. These elementary tests. while no doubt of distinct service, cannot aspire to rival an investigation which gives with moderate accuracy the percentage of total : nitrogen, the fractions of this existing as urea and as ammonia, and the actual amount of acetone. complex bodies present.

Further, when a fuller examination is undertaken, it is usually limited to abnormal cases. No clear comparison, therefore, is obtained between the normal and the ab. normal. It will be apparent later in this paper that it is not legitimate to judge of the ammonia percentage in pregnancy, normal or abnormal, from the percentage found in the urine of a non-pregnant individual.

This paper records the results of an investigation of the urine in 80 cases of pregnancy, both normal and toxic, and has as its special endeavour the description of sereral tests for that purpose. These have the particular merit of being applicable by a relatively inexperienced worker in a clinical, as distinguished from a bio-chemical, laboratory.

Mathison's Method of Ammonia E'stimation.

This test is performed as follows:

Shake for two minutes $25 \mathrm{c.cm}$. of urine with 15 grams (circe) of ncutrai potassium oxalate and $50 \mathrm{c}$.cm. distilled water. The A paper read in the Section of Obstetrics and Gynaecology at the 
oxalate clears the specimen by precipitating calcium salts and thereby makes the end-reaction more accurate. Neutralize with $\mathbf{N}, 10$ soda, using phenolphthalein as an indicator. Ald 5 c.em. neutralized commercial formalin. Titrate again with $\mathrm{N} 10$ sola till neutral. Falf an honr should elapse before the final reading is made. Each cubic centimetre equals 0.0014 gram of nitrogen in the urine as ammonia.

Formalin in the presence of ammonia forms urotropin, at the same time liberating the acid which was combined with the ammonia. If to neutralized urine be added neutralized formalin the indicator will show an immediate acidity, which can be measured by $\mathrm{N} / 10$ soda, and from which the ammonia content can be calculated.

The result is acknowledged to be sufficiently accurate foi clinical purposes, and the error in any pathological urine does not exceed 1 per cent. of the total nitrogenthat is, the reading is about 15 per cent. more than by Folin's method, which does not include the amino-acids. 'There is, howerer, no disadvantage from this inclusion of amino-acids.

Groat's Method of Nitrogen Estimation. The method of applying this test is as follows:

Add to 2 c.cm. urine 6 c.cm. concentrated sulphuri s acid and a few crystals of potassium sulphate in a Kjeldahl flask. Boil in a fumie cupboard till clear; cool, wash thoronghly into a larse flask, and estimate the total nitrogen as ammonium sulphate by the above method.

The neutralization of the washings can best be done with first 20 per cent., then 5 per cent., and finally 1 per cent. sodium hydroxicle, and if the nentral margin be passed a drop of acic should be rapidly added. Once asain the final reading represhould be rapidly added. Once again the final rexding represents a pink tint persisting for half an hour. The number of cubic centimetres of $\mathrm{N} / 10$ soda multiplied by 0.07 gives the slight modification of the original, and was kindly sent me by Dr. Groat in a private communication.

Groat's metlod, as will be seen, is an ingenious adaptation of Mathison's. The total nitrogen is converted to amnonium sulphute as in Kjeldahl's method, and thereafter the ammonia calculated as in Mahison's test. If a fume cupboard be not available, the long-necked flask can be fixed in a slightly opened window.

The method has the advantage over Kjeldahl's method in its simplicity, in the elimination of decinormal sulphuric acid solution, and in the fact that personal error is equalized for both Groat's and Mathison's tests by the similarity of the end results (phenolphthalein).

I have compared this method with Kjeldall's in a number of cases, and in none has the ammonia nitrogen percentage of the total nitrogen (calculated by each method) differed by more than 0.6 per cent. I consider, however, that it would be better for each worker to do some such comparison before relying entirely on Groat's method. The urine from fifteen non-pregnant cases, none suffering .from primary liver disease, in no case showed an ammonia coefficient above 5.5 per cent.

All striking results in the cases about to be mentioned were at once controlled and in every case confirmed by Kjeldahl's method.

Hart's Colorimetric Acidosis Index.

There have been several colorimetric estimations advocated in recent years for acetone complex bodies, but Hart's method, while perhaps less accurate than some of the others, is a very simple one and gives a reasonably accurate result.

Twó solutions are necessary:

(a) Ethyl aceto-acetate, 1 c.cm.; alcohol, 25 c.cm.; distilled rater, 1,000 c.cm

(b) Ferric chloride, 100 grams; distilled water, $100 \mathrm{c.cm}$.

Take two test tubes of equal ( 1 in.) calibre; in one put $10 \mathrm{c.cm}$. of $(a)$, and in the other $10 \mathrm{c.cm}$. of urine; add to each $1 \mathrm{c.cm}$. of $(b)$. Dilute the tube containing urine till the two match. The acidosis index per litre equals one-tenth of the rolume (cubic centimetres) in the tube of urine. To obtain the index proper (which represents the total acidosis estimated in terms of grams of $\beta$-oxybutyric acid), multiply the above by the number of litres passed in twenty-four hours

Quantities of acetone bodies insufficient to give a positive perchloride test but giving a sodium nitro-prusside reaction represent an acidosis index per litre of 0.5 .

By adding varying quantities of ethyl-aceto-acetate to norual urine, I have found that a very fair estimate of the amount added can be gauged by this simple test, and both in pregnancy and in other conditions it has proved both interesting and illuminating.
As regards qualitative tests for acetone bodies in the urine, Harding and Ruttan have recently shown conclit. sively that the ordinary sodium nitro-prusside-ammoniaacetic acid test (Lange or Le Nobel) is not alone a test for acetone, but detects as well diacetic acid - that this latter body, in fact, seems to augment the acetone reaction. They estimate the relative delicacy of Le Nobel's test and Gerhardt's test-that is, ferric chloride-as 30 to 7 . A urine, then, which only gives the former, may still contain diacetic acid. It must be remembered, too, that acetone appears first in orfer, and, with increase of these bodies, is accompanied by diacetic acid, and, later on, by $\beta$-oxy butyric acid. A positive diacetic acid test and a negative acetone reaction is, then, impossible, and it will be found that salicylates or their derivatives (such as diuretin) account for the phenomenon.

Discussion of the Possible Value of Data Obtained. (a) Tutal Nitrogen.

This figure should be used merely as the total from which to calculate the ammonia percentage. It can have no other reliable value unless the nitrogen. values of the food and faeces are simultaneously examined. This is particularly true in pregnancy, where there are very marked variations in the nitrogenous excretion at different periods. For instance, there is in the second half of pregnancy a tendency towards the retention of vitrogen it wust not, therelore, be immediately decided in late preguancy that a low total nitrogen value implicates inanition as a factor to be considered-say, in explanation of the presence of acetone bodies in the urine. The total nitrogen figure is, obviously, however, a vastly more useful figure than the urea nitrogen which, in toxic cases, varies inversely as the ammonia.

\section{(b) Ammonia Nitrogen.}

The normal coefficient in the non-pregnant state is 3 to 5 per cent.- that is to say, this percentage of the nitrogen in the urine is eliminated as ammonia. In pregnancy, even in clinically normal pregnancy, these limits may be greatly exceeded. There is a widespread and absolutely unjustifiable belief that this increase of ammonia must necessarily represent and measure an acidosis; but, as I have emplasized in an earlier paper, there is every reason to assign a part of the ammonia (and that perhaps a large one) to the histological changes occurring in the liver.

It is certain that primary liver disease-primary disturbance of the protein metabolism-is associated with an increase. Presumabl, he trea met ubolism has been interrupted half way by the $\mathbf{n}$ fif :ient functioning of degener ted cells, and ammonia the euy split off. To begin with, this shows that the liver eells are still-largely, at any rateliving, as otherwise this tirst stage of ammonia formation could not take place. The more widespread the degeneration the great $\mathbf{r}$ will be the ammonia formation.

It, will be at once apparent that if large areas of live substance in any disease have received a mortal blow they will be unable to functionate, and ammonia will, therefore, not $b e$ formed. In primary liver disease, then, the gravity of the injury is not directly proportionate to the increaso of ammonia once the borderland between degeneration and necrosis has been passed. There is a fundamental chemical difference between the two conditions, even althongh both are stages in the process of cell destruction.

In all cases the dependence on these two conditions of data obtained must be borne in mind. Thus, if a certain proportion of cells have altogether escaped - if, in other words, the lesion be mainly focal-there may be sufficient healthy tissue left to very considerably lessen the urinary evidences of the altered metabolism occurring in the affected parts.

Coming now to discuss the coefficient more particularly in relation to pregnancy, one is inevitably drawn to the conclusion that there is an assault on the liver which renders it classifiable as a primary liver disease. Detailed work, such as that of $E$ wing and Wolf, gives evidence that even in normal pregnancy there is a considerable strain on the liver-the margin of safety, no doubt, is a large one.

It is nuch to be regretted, too, that the post-mortein findings in fatal cases of toxic pregnancy liare not been more regularly compared with the urinary analyses during life.

In toxic pregnancy it is clear that increased ammonia is 
most in evidence in hyperemesis. While not wishing to dissociate unduly this form of toxic pregnancy from the eclamptic group, it is at the same time certain that in hyperemesis the lesion is essentially a degenerative one ; such necrosis as occurs appears late as the final stage of this degeneration. An increased ammonia coefficient, therefore, is what one would expect. It is just possible that in such a case, if necrosis supervened to any great extent, the ammonia might decrease towards the end, but I do not know of any reports of cases taken sufficiently often to have demonstrated this; yet in one rapidly fatal case (No. 12 in the series of Ewing and Wolf) the ammonia ratio was not increased at all, while the total nitrogen and the urea were both increased but relatively unchanged-a condition rather characteristic, as demonstrated by the experimental work of Jackson and Pearce, of a lesion where necrosis predominates.

On the other hand, in eclampsia the predominating lesion is often a massive focal necrosis which appears too suddenly and too early to be the last stage of the ordinary toxaemic degeneration, and, once more judging from experimental work, must follow a vascular obstruction. In such cases one would not expect the high ammonia values characteristic of vomiting, and, as a matter of fact, they do not occur. There may be early increase in mild cases, and, in general, in cases with a good prognosis-so far as one can give any prognosis in a disease where other factors, such as cerebral haemorrhage, may intervenebut otherwise there is but little.

Some pathological foundation will now become apparent for the empiric view of Whitridge Williams that, generally speaking, he regards a low ammonia value as a good sign in toxaemic vomiting and a bad sign in eclampsia. In persistent vomiting a low ammonia value would indicate a slighter degree of upset of the ammonia-urea proportion, or, pathologically interpreted, less degeneration; whereas ir eclampsia a low ammonia value would suggest that necrosis was very widespread, and a high one that a considerable proportion of liver cells was still alive and functioning, albeit imperfectly.

For a fuller comparison between the chemistry and histology of toxic pregnancy and these features as exhibited in comparative experimental work my earlier papers must be referred to. It should also be kept in view that the study of the ammonia variations in pregnancy is only one aspect of the changes taking place. The very important question of alterations in the rest-nitrogen, and in the amino-acid fraction of this cannot, unfortunately, be studied in purely clinical practice and must here be omitted; when such an attempt has been made the resilt usually indicates that this group las been made the dumping ground for all the errors in the other estimations:

\section{(c) Acidosis}

So far, I have merely indicated that variations in the ammonia value may be explained in pregnancy, as they certainly are explained in certain other conditions, by a primary disturbance of the protein metabolism.

We have now to decide, so far as is possible, to what extent an acidosis may take part in the alteration, and here at once one finds prevalent the most conflicting ideas. There is still a certain amount of mystery surrounding the whole question of acidosis and the criteria which different observers consider sufficient for its demonstration vary to an almost ridiculous extent. Some are content to judge absolutely by increased ammonia; this, as I have endeavoured to show, may be quite wrong. Others are content to label every case slowing some acetone in the urine as being of this nature; this is certainly a fallacy.

It will be best to discuss a few elementary points in relation to acidosis in general, and in so doing I will draw largely from the excellent work of Sellards.

As a preliminary it may be asked-Why does ammonia appear at all in normal urine? The usual answer given is that a slight grade of "physiological acidosis" exists under normal conditions; in other words, in order to assist in the nentralization of the acids derived from the food proteins and to establish an equilibrium of the acids and bases, even in the healthy state ammonia must be bound ta the former. If this be admitted, it would be possible to conjecture a condition which would be pathological merely in degree.
Now, in definitcly pathological conditions, acidosis may be considered under two headings-namely:

1. There is a qualitative change.-This is usually indicated by the appearance in the urine of salts of certain well-known foreign acids. It must not be forgotten, however, that even in diabetes these recognized foreign acids may not be the sum total of the unusual acid bodies present. It is also possible to imagine, and there is considerable evidence in favour of the view, that there are types of acidosis in which the better-known acids-the acetone-complex bodies-do not appear at all. This is particularly so in cases of nephritis, especially in uraemia or cases tending thereto. The remarkable alleviation of symptoms which can occur in some of these by administering sodium bicarbonate will be a revelation to those who have not tried it.

2. There is a quantitative change.-The essential in this is a decreased amount of fixed bases in the tissues. It cannot be too strongly asserted that symptoms of pathological acidosis are not due to the acid bodies present but to a diminution of fixed bases in the tissues as the result of an attempt to neutralize the acids. Now, at a certain point-although by no means necessarily at a very early stage - a compensatory increast of ammonia production is induced in order to spare the fixed bases. Ammonia, however, is by no means the only compensatory mechanism, and an acidosis may occui without mucl disturbance of the coefficient. Other bases, such as calcium, magnesium, potassium, and sodium, take their share, particularly in extreme cases. In herbivorons animals these, of course, take an early and important part in the neutralization.

What, then, is the best test that the body is suffering from the condition ordinarily called " acidosis" - that is, a depletion of the fixed bases? It would appear very reasonable to believe that the best test is one of tolerance to alkali. . Expressed differently, if the body is in no need of fixed bases, any alkali introduced will be eliminated, and the urine will becone alkaline. On the other hand; if there be a need for fixed bases, when alkali is introduced, it will remain in the body, and the reaction of the urine will remain unchanged.

I am sorry to see that Dr. Herbert Williamson is not very enthusiastic over this test. To me it appears-theoretically, at least--a more important and reliable indicator than either a high ammonia value or the demonstration of acetone-complex bodies in the urine. Its absolute value can only be determined when a long series of cases of normal and toxic pregnancy, and of other conditions, has been put to the test. Meanwhile, it has the merit of being simple in execution ( 5 grams of sodium bicarbonate every six hours per os, or, in cases with gastric upset, the intravenous injection of 2 to 4 per cent. solutions), and is to some extent a quantitative as well as a qualitative indicator-that is, one can judge of the degree of depletion in inverse proportion to the ease with which an alkaline reaction can be produced and maintained.

There are, then, two reasons for the appearance of increased ammonia in the urine:

1. From a disorder of the primary protein metabolism.

2. As a purely compensatory process.

In the latter case the increase will disappear if sufficient sodium bicarbonate be given; in the former case it will not. We must differentiate, therefore, in pregnancy between these two mechauisms, and one must not attribute increased ammonia to an acidosis until one has eliminated the possibility of primary metabolic error. Williamson's observations have not convinced him that the ammonia coefficient can be regarded as an index of the gravity of the hepatic lesions, for he finds that after the administration of sodium bicarbonate the ammonia falls. Personally, I have not found this so in all cases, but, owing to lack of facilities, the number in which I have had the opportunity of trying it has been small. Sellards con. firms this by quoting an instructive case in which a rery high coefficient, acetone complex bodies in only slight amount, and a normal tolerance to alkali occurred in a case of toxaemic pregnancy. This is not explicable on the supposition that the ammonia increase was purely com. pensatory:

In definite acidosis, too, of the ordinary type, the ammonia coefficient does not rise till the acid bodies are well in 
evidence in the urine; that is to say, the fixed bases of the body have done the earlier stages of neutralization.

In general, then, the excretion of ammonia in pregnancy is not a reliable basis for the detection or estimation of acidosis, for it may be normal in acidosis and high without acidosis. It is really more important than might at first be thought to make certain of the extent of an acidosis from a practical point of view. A rery frequent procedure in toxic pregnancy is copious bleeding; if the fixed bases are already depleted this will still further lessen them, and will only be justifiable if alkali be immediately added.

If one caunot be certain of an acidosis by an estimation of the ammonia, does an estimation of the acetone-complex botlies give a better indication? On the whole, I think that it does.

As I have explained, there may be other acids than those so readily recognized as taking part in the process, but where the acidosis is of the type wherein these bodies are excreted in quantity, and where, as in diabetes, there is no reason to hypothecate a primary disturbance in the nuea metabolism, we know that these acid bodies increase with the clinical symptoms of acidosis and that there is a lefinite relationship between the imminence of coma and the quantity of those demonstrable. In pregnancy, then, it appear's likely that their estimation may give an index although probably a very rough one, of the degree of acidosis. Even here it may be necessary to make some reservation and to draw a distinction between acidosis and acid intoxication, for it is undoubted that individuals, both pregnant and non-pregnant, occasionally excrete remarkable quantities of these bodies with little or no general upset.

The results I am about to record are of interest more particularly in relation to symptomless pregnancy, but liave also some value in a discussion of toxic conditions.

Collection and Preservation of Specimens.

Mixed specimens were not considered necessary nor, in toxic cases, even advisable. Repeated tests in pregnant cases and in non-pregnant individuals, normal and abucemal, showed that the percentage of ammonia nitrogen to total nitrogen did not vary more than 1 per cent. in specimens from different periods of the twentyfour hours. In acutely toxic cases, on the other hand, the difference within a few hours has been found at times to be very marked, and it appears to me to be important to take specimens as often as possible rather than the single result from a twenty-four hours' mixed specimen. Particularly is this important from a prognostic point of view, as, in any one case, the direction of the variation should carry more weight than the actual increase of ammonia.

Accordingly, the method adopted was to shake with thymol ciystals each specimen directly it was passed. Experiment showed that there was a definite increase in the coefficient within a relatively short time if this were omitted.

\section{Results.}

The cases examined number 80 , and the following are the results :

\section{Symptomless Pregnancy.}

I wish to draw your attention more particularly to these cases. Nearly all these cases were examined on more than one occasion, and the majority of the readings were taken during the last six weeks of pregnancy.

The anmonia coefficient was found in 66 cases to range from 2.2 per cent. to 12 per cent. These results are scarcely in accordance with those of Williamson, who gives readings of 4 to 5 per cent. They are, however, on a par with those obtained by Ewing and Wolf, whose figures show in clin cally normal pregnancy a coefficient of 2.8 to 10.8 per cent.

In my own ca-es, in 72 per cent. the coefficient was over 5.5 per cent., in 20 per cent. it was over 8 per cent. It was noted that there was a definite tendency for it to become higher as pregnancy advanced, and to be highest in labour. In no case was a coefficient over 5.5 per cent. obtained before the fifth month.

'Iwo of the many cases examined during labour had particularly high readings, 13 per cent. and 17 per cent. 'These results were at once controlled by Kjeldall's method. There was neither albumin nor acetone bodies in either case and the index dropped very rapidly when labour was over.

Two cases in labour showed the presence of acetone bodies totailing an index per litre of 1.5 and 1 respectively. The corresponding coefficients were 9 per cent. and 10 per cent. In one of these the tolerance test for alkali was done, but no evidence of retention was made out and the coefficient did not fall.

In four cases of late pregnancy, giving high ammonia values ( 9 to 12 per cent.) withont acetone bodies, the tolerance test was carried out; in none was there any difficulty in rendering the urine alkaline.

A trace of albumin was found in 9 of these 66 cases, but bore no relation to a high ammonia coefficient.

Pregnancy with Symptoms.

As I have stated, the details of these cases are not as full as I could wish, but I think the figures given in several cases do show that Whitridge Williams is correct in corelating morely closely than he was at first disposed to do, from the points of view both of diagnosis and prognosis, the clinical with the urinary condition.

\section{Hyperemesis.}

One acute case was observed for a week (3 tests) during which the coefficient, temperature and pulse remained normal. There was no acetone or albumin. The patient was two and a half months pregnant, and, as the vomiting showed no tendency to become better under medicinal treatment, rectal feeds (glucose with milk pancreatized for twenty-four hours as directed by Rendle Short) were given and retained well. The vomiting appeared to im. prove during the next two or three days, yet the patient began to look ill, the pulse became fast and the tempera. ture heightened. During the next two days the coefficient rose to 15 per cent. and then 25 per cent. Acetone and diacetic acid appeared in the urine, but unfortunately were not estimated.

There was greatly increased tolerance to sodium bicarbonate without reduction of the coefficient. The uterus was emptied, with an immediately successful result.

A second case developed vomiting about the fifth month with a little irregular temperature and moderate albumin. There was a very slight deposit of pus in the urine, which may have accounted for the temperature but certainly did not account for the albumin. The coefficient was 4.9 per cent., and the patient was put on calcium lactate.

One week later, althougl the patient appeared improved, bad less vomiting, and was retaining food well, there. appeared acetone bodies with an index per litre of 4 and an ammonia coefficient of 20.3 per cent. Allkali given as a tolerance test and as a therapeutic measure reduced the coefficient to 9.2 per cent. I decided to do nothing radical, and was justified in the result.

Other cases, more moderate in character, developed coefficients of 10 per cent. and 11.8 per cent. In the latter there were no acetone bodies, but in the former (third month) an index per litre of 1.7 was given and remained so for a few days, when spontaneous abortion took place.

\section{Eclampsia.}

Five cases were observed. In one there was an acidosis index per litre of 2.2; the other four were negative. This agrees with the results of Ewing and Wolf, who found acetone bodies present in one case and absent in three. The coefficients ranged between 13 to 18 per cent. in four cases. They were all in labour at the time, and they cannot, therefore, be said to have exhibited any very marked rise. After labour, in three of these the index came to normal very rapidly. In the fourth mental symptoms developed and the ratio remained high for one week, subsiding gradually with a return to normal cerebration.

The tifth case was a fatal one. The index was examined on admission and the coefficient found to be 8.5 per cent.; the urine in the bladder at death-six hours later-gave a reading of 6 per cent. There was only slight albumin (0.05 Esbach) and there was no acetone. No opportunity was given for a tolerance test. The post-mortem examination showed that the patient had died of cerebral haemor. rhage. There was, however, a very marked necrosis in the liver of the type characteristic of the disease, and it is of interest that this should have shown a lower coefficient than any of the otheros. 
3. Pre-eclamptic State.

Two cases examined in the ninth month with moderate albumin showed coefficients of 113 and 10.4 per cent. and no acetone. T'lese figures, it will be noted, give less indication of the patient's condition than did the clinical examination with obvious oedema, headuche, and scanty loaded urine.

4. Nephritis in Pregnancy.

One case was seen in the seventh month. The urine was scanty and showed a coefficient of 3.5 per cent., albumin 0.7 (Esbach), and no acetone. There was moderate oedema and considerable breathlessness. The tolerance test showed that alkali was retained in the body. In spite of the low coefficient and the absence of acid bodies, it seems perfectly justifiable to class this case as one of acidosis, The patient was spontaneously delivered of a living child thirty-six hours after the observations were made, and died of uraemia a few days afterwards.

A second case was also in the seventh month. She was heavily waterlogged and showed albuminuric retinitis The urine showed no acetone but gave a coefficient of 16.4 per cent., and albumin 1 (Esbach); tolerance to alkali was nornal; labour was induced by bougies and three days later the coefficient was 9.1 per cent., and a week later 4 per cent. The albumin and oedenia rapidly decreased.

\section{Toxacmia of Doubtful Origin.}

This case was seen at the fourth month. She had headache and temperature, with drowsiness and occasional vomiting. The ammonia coefficient proved to be 5 per cent.; there were no acetone bodies, and the tolerance test was normal. One was led, therefore, to look elsewhere than in the pregnancy for the cause, and a Widal reaction and the subsequent history, which included miscarriage, proved the condition to be typhoid fever.

In conclusion, I frankly admit that I feel scarcely more confidence in my interpretation of many of these results than. I do in an interpretation of acidosis in general. My main object is to excite discussion and to stimulate a little more interest in the whole question, more particularly in maternity hospitals, where series of valuable data could readily be compiled.

It would be of great interest to see what parallelism cxists between tolerance to alkali and the other criteria of acidosis. Only then will it be possible to determine to whas extent acidosis represents an intoxication.

In general I think that one is justitied, (1) so far as the practical aspect is concerned, in endorsing Whitridge Williams's considered view that only by a close comparison of the clinical condition with the urinary findings can one judge of degrees of gravity; and (2), so far as academic research is concerned, in keeping a very open mind on the subject till more figures are available.

\section{TBBLIOGRAPHY}

Williams, Journ. Obstet. and Gyn. Brit. Emp;, November, 1912. Mathison, British MEDICAL JoUkNaL, March 20th, 1909. Groat, Amer Journ. Obstet, December, 1911. Hart. Quart. Journ. Meat, Auly, 1912 Harding and Ruttan, Biochem. Jinurn., October, 1912.
Jackson and Pearce, Journ. Exper. Med., vol ix, 1907. Pearce, Journ. Medl. Reseavch, vol. xii, 1904 ; ibid., vol. xiv, 1906; Journ. Exper. Med., ol. viii, 1906. 'Ewing and Wolt, Amer. Joum. Obstet., vol. iv, 1907 . Sellards, Bull. Johns Hopkinss Hosp., October, 1912, April and May, 1914. Gun. Brit. Emp. Oct

THE British Fire Prevention Committee (8, Waterloo Place. London, S.W.), having found that deaths had been caused by the bursting of chemical liquid fire extinguisiners, and being satisfled that some of the appliances sold were of inferior quality, issued some time aso a pro. visional specification. It has' now replaced this by a definite specification, copies of which, together with lists of appliances complying with it, will be forwarded in response to a written application, accompanied by a stamped foolscap envelope. The committ e states that its requirements are not so severe as to handicap legitimate endeavours of the trade, and also that "Portable liquid fire extinguishers are not the only form of portable first-aid fire appliances, but that ordinary buckets of water (together with hand pumps) are equally, if not more, effective in the majority of cases, and, further, have considerable economical advantages for ordinary household, office, shop, and factory purposes."

\section{CASES OF INOPERABLE CANCER TREATED} WITH GOAT SEREM.:

\author{
Bi ALBERT WILSON, M.D.,
} LONDON.

THER: has always been a great mystery about the blood in malignant disease. Why, for instance, should cancer remain stationary in one patient, or even disappear spon. taneously, and progress in others? Why should one species of animal be apparently immune to cancer, and? why should inoculation of one animal of the same species with cancer give rise to tumour growth and the same not occur in another?

Attacking the problem from the blood point of view, Dr. J. A. Shaw-Mackenżie ${ }^{1}$ has shown that while normal serum in health contains a substance which has the property of activating or accelerating the fat-splitting action of pancreatic juice and pancreatic extracts, in certain cases of cancer this property is considerably increased. In progressive and advanced cases this power of the serum is decreased compared with the action of normal serum examined at the same time as a control, while the normal antitryptic action of the serum is increased in accordance with the original observations of Marcus, Brieger, and Trebing. When these two reactions are present they aid in the diagnosis of cancer, and when they are absent they exclude cancer.

During quiescence or improvement or after apparent recovery the accelerating fat-splitting action of the serum is high, while the antitryptic action falls to normal or is subnormal.

This accelerating fat-splitting property of the serum is greater in the lower animals than in human serum, while the antitryptic action of the serum is not greater than with normal human serum. Inoculation of animals with normal tissues or with cancer tissue is followed by a similar in crease in this property of the blood; while in mice which had been inoculated with mouse tumour and did not "take," and also in mice which had recovered spontaneously from large growths, the accelerating fat splitting action of the serum is high.

In view of such facts Shaw-Mackenzie has suggested that "the fat-splitting acceleration is a possible and natural factor in resistance to disease in carcinoma and in other conditions," and "if so, serum and substances which increase this action or protective mechanism are indicated in treatment."

In illustration Dr. Shaw-Mackenzie records the results of examination of the blood in two cases of apparent re. covery from inoperable cancer, examination of the blood having been made before, during, and after treatment with injections of the patients own serum, together with the administration of bile salts by the mouth. In one case the figures rose from 8.6 before treatment to 14.7 after treatment; while the antitryptic action fell from 7 to 4 . This was a case of inoperable breast cancer in March, 1911. The patient is now (June, 1914) in very good health generally, but a small lump remains locally.

In the other case-one of inoperable uterine cancer-the figures rose from 10.1 before treatment to 14.2 after, whilo the antitryptic action fell fron 6 to 3 . This patient is in good health, now $2 \frac{3}{4}$ years after treatment.

In general terms, animal serum is higher in the fat. splitting, accelerating, or activating property than normal human serum. 'This is witnessed in Dr. Shaw-Mackenzie's observation, namely, that the fat-splitting action of the serum in the goat was 14.5 compared with normal human serum, which I understand from him varies considerably in health, but approximately averages 10.0

The treatment of inoperable cancer, therefore, by normal goat serum is not empirical, for obvionsly we are introducing an activator of the fat-splitting protectire action in the tissues.

In 1890 a patient, aged 60, suffering from inoperable cancer of the uterus was sent to me by Dr. Robert Barnes. It occurred to me to inject goat serum, as the goat was considered by veterinary surgeuns to be immune to cancer. A healthy goat was procured and $6 \mathrm{oz}$. of serum were injected in the *Abridged from a paper read in the Section of Pharmacologs,
Therapentics, and Dietetics at the Annual Medical Association a the British 